\title{
Cloaking and Shielding Objects in a Fluid Flow
}

\author{
By injecting momentum into the fluid around an object, researchers can \\ freely switch between obscuring the object's presence and canceling \\ hydrodynamic forces on it.
}

\section{By Erika K. Carlson}

A mong the best-known technologies deployed by Star Trek's starships are their invisibility-inducing cloaking devices and their shields. In real life, a limited form of invisibility can be achieved passively, using metamaterials. Now, Evgeniy Boyko of the Technion - Israel Institute of Technology and colleagues have designed an active method that incorporates shields, too. Their technique works for an object sitting in a fluid flow and lets them switch between "cloaking" and "shielding" modes at will [1].

Hydrodynamic cloaking works by obscuring the effects that an object has on fluid flowing around it. A metamaterial with a certain structure can, for example, deflect flow around the object to alter its interaction with the structure (see Synopsis: Hydrodynamic Cloaks).

The new method of Boyko and colleagues uses a different approach. Instead of using passive structures, they actively

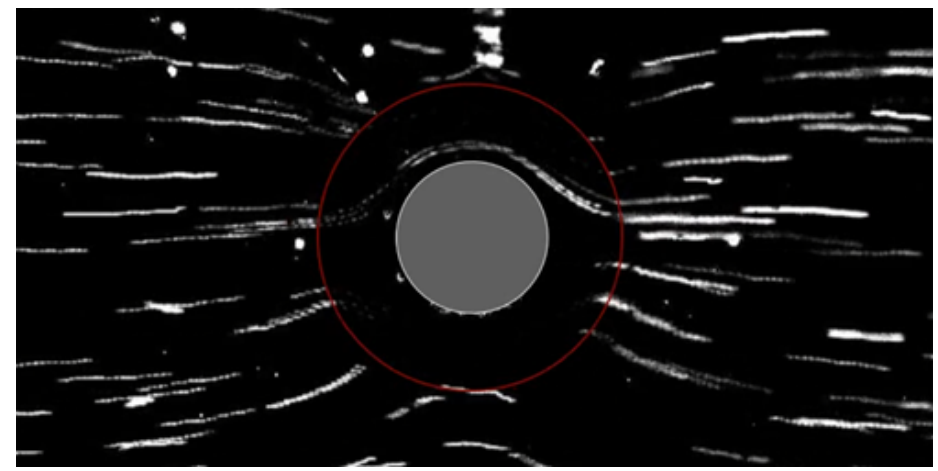

Credit: V. Bacheva/Technion - Israel Institute of Technology; IBM Research Europe
Video 1: The team's active approach allows them to switch between "cloaking" and "shielding" an object from a fluid flow at will.

Credit: E. Boyko et al. [1]

inject momentum into the fluid using a mechanism called electro-osmosis. This technique lets them control the surrounding fluid's momentum via electric fields so that, in the cloaking mode, the flow outside of the cloaked region gives no hint of the object's presence. The researchers can also switch to manipulating the fluid flow around the object such that any hydrodynamic forces that the flow would exert on the object are eliminated. They call this cancellation of forces the shielding mode.

The team describes the approach theoretically for a range of object shapes in a microscale flow and demonstrates it experimentally in a microfluidic chamber. The technique could have a range of applications, the researchers say, including acting as "tweezers" to hold soft objects-such as cells-stationery in a fluid flow.

Erika K. Carlson is a Corresponding Editor for Physics based in New York City.

\section{REFERENCES}

1. E. Boyko et al., "Microscale hydrodynamic cloaking and shielding via electro-osmosis," Phys. Rev. Lett. 126, 184502 (2021). 